\title{
From prescription to over-the-counter: A Conversation Analytic single case study of treatment recommendations in general practice
}

\author{
JOHANNA LINDELL
}

Antibiotic overuse and resulting antibiotic resistant bacteria are a growing problem both nationally and globally (ECDC 2014). Primary care physicians prescribe $90 \%$ of the antibiotics used for humans in Denmark, and $2 / 3$ of these are prescribed for upper respiratory tract infection (Bjerrum et al. 2014). However, many of these prescriptions are unnecessary and excessive. The reasons for overprescription of antibiotics are many, but this article focuses only on one: the importance of language for the treatment recommendation and its acceptance or rejection. Looking at a single Danish doctor-patient conversation which moves successfully from a recommendation for antibiotics to a recommendation for non-antibiotic nasal spray, it is suggested that the format of the treatment recommendation may influence patient willingness to bypass antibiotics. Using Conversation Analysis, the two treatment recommendations (one for antibiotics and a subsequent one for nasal spray) are analyzed in detail, and the reasons for the acceptance of the second one over the first one are discussed. Designing the treatment recommendation as an option which the patient is given agency to choose is suggested as a possible way to reverse or avoid antibiotic prescription in cases where it is medically safe. 